\title{
HODGE THEORY IN COMBINATORICS
}

\author{
MATTHEW BAKER
}

\begin{abstract}
If $G$ is a finite graph, a proper coloring of $G$ is a way to color the vertices of the graph using $n$ colors so that no two vertices connected by an edge have the same color. (The celebrated four-color theorem asserts that if $G$ is planar, then there is at least one proper coloring of $G$ with four colors.) By a classical result of Birkhoff, the number of proper colorings of $G$ with $n$ colors is a polynomial in $n$, called the chromatic polynomial of $G$. Read conjectured in 1968 that for any graph $G$, the sequence of absolute values of coefficients of the chromatic polynomial is unimodal: it goes up, hits a peak, and then goes down. Read's conjecture was proved by June Huh in a 2012 paper making heavy use of methods from algebraic geometry. Huh's result was subsequently refined and generalized by Huh and Katz (also in 2012), again using substantial doses of algebraic geometry. Both papers in fact establish log-concavity of the coefficients, which is stronger than unimodality.

The breakthroughs of the Huh and Huh-Katz papers left open the more general Rota-Welsh conjecture, where graphs are generalized to (not necessarily representable) matroids, and the chromatic polynomial of a graph is replaced by the characteristic polynomial of a matroid. The Huh and HuhKatz techniques are not applicable in this level of generality, since there is no underlying algebraic geometry to which to relate the problem. But in 2015 Adiprasito, Huh, and Katz announced a proof of the Rota-Welsh conjecture based on a novel approach motivated by but not making use of any results from algebraic geometry. The authors first prove that the Rota-Welsh conjecture would follow from combinatorial analogues of the hard Lefschetz theorem and Hodge-Riemann relations in algebraic geometry. They then implement an elaborate inductive procedure to prove the combinatorial hard Lefschetz theorem and Hodge-Riemann relations using purely combinatorial arguments.

We will survey these developments.
\end{abstract}

\section{INTRODUCTION}

A sequence $a_{0}, \ldots, a_{d}$ of real numbers is called log-concave if $a_{i}^{2} \geq a_{i-1} a_{i+1}$ for all $i$. Many familiar and naturally occurring sequences in algebra and combinatorics, such as the sequence of binomial coefficients $\left(\begin{array}{l}n \\ k\end{array}\right)$ for $n$ fixed and $k=0, \ldots, n$, are log-concave. A generalization of the log-concavity of binomial coefficients is the fact (attributed to Isaac Newton) that if the polynomial $\sum_{k=0}^{d} a_{k} x^{k}$ has only real zeros, then its sequence of coefficients $a_{0}, \ldots, a_{d}$ is log-concave. A useful property of log-concave sequences of positive real numbers is that they are unimodal, meaning that there is an index $i$ such that

$$
a_{0} \leq \cdots \leq a_{i-1} \leq a_{i} \geq a_{i+1} \geq \cdots \geq a_{d}
$$

Received by the editors May 15, 2017.

2010 Mathematics Subject Classification. Primary 05B35, 58A14.

The author's research was supported by the National Science Foundation research grant DMS1529573. 
Log-concavity also plays an important role in probability and statistics, as well as in applications of fields such as econometrics. As a prototypical example, normal distributions (as well as many other commonly occurring probability distributions) are log-concave. The convolution of two log-concave probability distributions on $\mathbb{R}^{d}$ is again log-concave. Moreover, under suitable technical hypotheses, a probability measure is log-concave if and only if its density function is log-concave. The class of log-concave distributions can thus be viewed as a naturally occurring and useful enlargement of the class of all normal distributions (see [26] for further discussion).

Log-concavity also occurs naturally in convex geometry and algebraic geometry. For example, the celebrated Aleksandrov-Fenchel inequality states that for any two convex bodies $A, B$ in $\mathbb{R}^{n}$, the sequence

$$
V_{0}(A, B), V_{1}(A, B), \ldots, V_{n}(A, B)
$$

of mixed volumes is log-concave. And the Hodge index theorem and its various generalizations (about which we will say more below) can be viewed as log-concavity statements about certain intersection numbers on smooth projective algebraic varieties.

Our goal in this paper will be to survey some recent mathematical breakthroughs establishing the log-concavity of certain naturally occurring sequences in matroid theory (see section 3 for an introduction to matroids). The genesis of this line of investigation was Read's 1968 conjecture that for any graph $G$, the sequence of absolute values of coefficients of the chromatic polynomial is unimodal; it was later conjectured by Hoggar that the sequence is in fact log-concave. Read's conjecture (along with the stronger log-concavity statement) was proved in 2012 by June Huh using methods from algebraic geometry. Huh's result was subsequently refined and generalized by Huh and Katz, again using substantial doses of algebraic geometry.

The breakthroughs of Huh and Huh-Katz left open the more general RotaWelsh conjecture, where graphs are generalized to matroids and the chromatic polynomial of a graph is replaced by the characteristic polynomial of a matroid. The Huh and Huh-Katz techniques are not applicable in this level of generality, since there is no underlying algebraic geometry to which to relate the problem. But in 2015 Adiprasito, Huh, and Katz announced a proof of the Rota-Welsh conjecture based on a novel approach motivated by but not making use of any results from algebraic geometry. The authors first prove that the Rota-Welsh conjecture would follow from combinatorial analogues of the hard Lefschetz theorem and Hodge-Riemann relations in algebraic geometry. They then implement an elaborate inductive procedure to prove the combinatorial hard Lefschetz theorem and Hodge-Riemann relations using purely combinatorial arguments.

We will survey these developments, as well as a recent breakthrough of Huh and Wang establishing the top-heavy conjecture of Dowling and Wilson for matroids representable over some field. Their result (whose proof again makes use of deep results from algebraic geometry) can be seen as a vast generalization of the de Bruijn-Erdős theorem that every noncollinear set of points $E$ in a projective plane determines at least $|E|$ lines. 


\section{UNIMODALITY AND LOG-CONCAVITY}

A sequence $a_{0}, \ldots, a_{d}$ of real numbers is called unimodal if there is an index $i$ such that

$$
a_{0} \leq \cdots \leq a_{i-1} \leq a_{i} \geq a_{i+1} \geq \cdots \geq a_{d} .
$$

There are numerous naturally occurring unimodal sequences in algebra, combinatorics, and geometry. For example:

Example 2.1 (Binomial coefficients). The sequence of binomial coefficients $\left(\begin{array}{l}n \\ k\end{array}\right)$ for $n$ fixed and $k=0, \ldots, n$ (the $n$th row of Pascal's triangle) is unimodal.

The sequence $\left(\begin{array}{l}n \\ 0\end{array}\right),\left(\begin{array}{l}n \\ 1\end{array}\right), \ldots,\left(\begin{array}{l}n \\ n\end{array}\right)$ has a property which is in fact stronger than unimodality: it is log-concave, meaning that $a_{i}^{2} \geq a_{i-1} a_{i+1}$ for all $i$. Indeed,

$$
\frac{\left(\begin{array}{l}
n \\
k
\end{array}\right)^{2}}{\left(\begin{array}{c}
n \\
k-1
\end{array}\right)\left(\begin{array}{c}
n \\
k+1
\end{array}\right)}=\frac{(k+1)(n-k+1)}{k(n-k)}>1 .
$$

It is a simple exercise to prove that a log-concave sequence of positive numbers is unimodal.

Some less trivial, but still classical and elementary, examples of log-concave (and hence unimodal) sequences are the Stirling numbers of the first and second kind.

Example 2.2 (Stirling numbers). The Stirling numbers of the first kind, denoted $s(n, k)$, are the coefficients which appear when one writes falling factorials $x \downarrow_{n}=$ $x(x-1) \cdots(x-n+1)$ as polynomials in $x$,

$$
x \downarrow_{n}=\sum_{k=0}^{n} s(n, k) x^{k} .
$$

This sequence of integers alternates in sign. The signless Stirling numbers of the first kind $s^{+}(n, k)=|s(n, k)|=(-1)^{n-k} s(n, k)$ enumerate the number of permutations of $n$ elements having exactly $k$ disjoint cycles.

The Stirling numbers of the second kind, denoted $S(n, k)$, invert the Stirling numbers of the first kind in the sense that

$$
\sum_{k=0}^{n} S(n, k)(x)_{k}=x^{n} .
$$

Their combinatorial interpretation is that $S(n, k)$ counts the number of ways to partition an $n$ element set into $k$ nonempty subsets.

For fixed $n$ (with $k$ varying from 0 to $n$ ), both $s^{+}(n, k)$ and $S(n, k)$ are logconcave and hence unimodal.

Another example, proved much more recently through a decidedly less elementary proof, concerns the sequence of coefficients of the chromatic polynomial of a graph. This example will be the main focus of our paper.

Example 2.3 (Coefficients of the chromatic polynomial). Let $G$ be a finite graph 1 In 1912, George Birkhoff defined $\chi_{G}(t)$ to be the number of proper colorings of $G$ using $t$ colors (i.e., the number of functions $f: V(G) \rightarrow\{1, \ldots, t\}$ such that $f(v) \neq f(w)$ whenever $v$ and $w$ are adjacent in $G)$, and he proved that $\chi_{G}(t)$ is a polynomial in $t$, called the chromatic polynomial of $G$.

\footnotetext{
${ }^{1}$ We allow loops and parallel edges.
} 


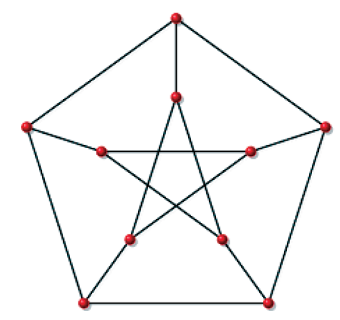

Figure 1. The Petersen graph

For example 2 if $G=T$ is a tree on $n$ vertices, then the chromatic polynomial of $G$ is

$$
\chi_{T}(t)=t(t-1)^{n-1}=\sum_{k=1}^{n}(-1)^{n-k}\left(\begin{array}{l}
n-1 \\
k-1
\end{array}\right) t^{k} .
$$

If $G=K_{n}$ is the complete graph on $n$ vertices, then

$$
\chi_{K_{n}}(t)=t(t-1) \cdots(t-n+1)=\sum_{k=1}^{n} s(n, k) t^{k} .
$$

And if $G$ is the Petersen graph, depicted in Figure 1, then $\chi_{G}(t)=t^{10}-15 t^{9}+105 t^{8}-455 t^{7}+1353 t^{6}-2861 t^{5}+4275 t^{4}-4305 t^{3}+2606 t^{2}-704 t$.

Ronald Read conjectured in 1968 that for any graph $G$ the (absolute values of the) coefficients of $\chi_{G}(t)$ form a unimodal sequence, and a few years letter Stuart Hoggar conjectured that the coefficients in fact form a log-concave sequence 3 Both conjectures were proved only relatively recently by June Huh [17.

Another interesting and relevant example concerns linearly independent sets of vectors:

Example 2.4. Let $k$ be a field, let $V$ be a vector space over $k$, and let $A$ be a finite subset of $V$. Dominic Welsh conjectured that $f_{i}(A)$ is a log-concave sequence, where $f_{i}(A)$ is the number of linearly independent subsets of $A$ of size $i$. For example, if $k=\mathbb{F}_{2}$ is the field of two elements, $V=\mathbb{F}_{2}^{3}$, and $A=V \backslash\{0\}$, then

$$
f_{0}(A)=1, \quad f_{1}(A)=7, \quad f_{2}(A)=21, \quad f_{3}(A)=28 .
$$

This conjecture is a consequence of the recent work of Huh and Katz [18] (cf. 21]).

Finally, we mention an example of an apparently much different nature coming from algebraic geometry:

Example 2.5 (Hard Lefschetz theorem). Let $X$ be an irreducible smooth projective algebraic variety of dimension $n$ over the field $\mathbb{C}$ of complex numbers, and let $\beta_{i}=\operatorname{dim} H^{i}(X, \mathbb{C})$ be the $i$ th Betti number of $X$. (Here $H^{*}(X, \mathbb{C})$ denotes the singular cohomology groups of $X$.) Then the two sequences $\beta_{0}, \beta_{2}, \ldots, \beta_{2 n}$ and

\footnotetext{
${ }^{2}$ Looking at the analogy between the formulas $\chi_{T}$ and $\chi_{K_{n}}$, and between $s(n, k)$ and $S(n, k)$, it may be reasonable to think of $(-1)^{n-k}\left(\begin{array}{l}n \\ k\end{array}\right)$ as a binomial coefficient of the first kind and of the usual binomial coefficients as being of the second kind. This fits in neatly with the inversion formulas $(1+x)^{n}=\sum\left(\begin{array}{l}n \\ k\end{array}\right) x^{k}$ and $x^{n}=\sum(-1)^{n-k}\left(\begin{array}{l}n \\ k\end{array}\right)(1+x)^{k}$.

${ }^{3} \mathrm{Log}$-concavity implies unimodality for the coefficients of $\chi_{G}(t)$ by the theorem of Rota mentioned at the end of section 4.2
} 
$\beta_{1}, \beta_{3}, \ldots, \beta_{2 n-1}$ are symmetric and unimodal. Moreover, this remains true if we replace the hypothesis that $X$ is smooth by the weaker hypothesis that $X$ has only finite quotient singularities, meaning that $X$ looks locally (in the analytic topology) like the quotient of $\mathbb{C}^{n}$ by a finite group of linear transformations.

The symmetry of the $\beta_{i}$ 's is a classical result in topology known as Poincaré duality. And one has the following important strengthening (given symmetry) of unimodality: there is an element $\omega \in H^{2}(X, \mathbb{C})$ such that for $0 \leq i \leq n$, multiplication by $\omega^{n-i}$ defines an isomorphism from $H^{i}(X, \mathbb{C})$ to $H^{2 n-i}(X, \mathbb{C})$. This result is called the hard Lefschetz theorem. In the smooth case, it is due to Hodge; for varieties with finite quotient singularities, it is due to Saito, and it uses the theory of perverse sheaves.

For varieties $X$ with arbitrary singularities, the hard Lefschetz theorem still holds if one replaces singular cohomology by the intersection cohomology of Goresky and MacPherson (cf. [10]).

Surprisingly, all five of the above examples are in fact related. We have already seen that Example 2.1, as well as Example 2.2 in the case of Stirling numbers of the first kind, are special cases of Example 2.3. We will see in the next section that Examples 2.3 and 2.4 both follow from a more general result concerning matroids. And the proof of this theorem about matroids will involve, as one of its key ingredients, a combinatorial analogue of the hard Lefschetz theorem (as well as the Hodge-Riemann relations, about which we will say more later).

\section{Matroids}

Matroids were introduced by Hassler Whitney as a combinatorial abstraction of the notion of linear independence of vectors. Matroid theory has since become ubiquitous in combinatorics, in part because matroids can be viewed (in a certain precise sense) as generalizing graphs. They can also be viewed as combinatorial geometries, generalizing configurations of points, lines, planes, etc., in projective spaces. Matroid theory has applications to a diverse array of fields including geometry, topology, combinatorial optimization, network theory, and coding theory. Among the many aspects of matroid theory that we will unfortunately not have space to delve into, we mention the surprising and useful fact that matroids can be used to characterize when the greedy algorithm gives an optimal solution to a suitable class of problems.

Our primary references for matroid theory are [25] and [28].

3.1. Independence axioms. There are many different (cryptomorphic) ways to present the axioms for matroids, all of which turn out to be nonobviously equivalent to one another. For example, instead of using linear independence, one can also define matroids by abstracting the notion of span. We will give a brief utilitarian introduction to matroids, starting with the independence axioms.

Definition 3.1 (Independence axioms). A matroid $M$ is a finite set $E$ together with a collection $\mathcal{I}$ of subsets of $E$, called the independent sets of the matroid, such that:

(I1) The empty set is independent.

(I2) Every subset of an independent set is independent.

(I3) If $I, J$ are independent sets with $|I|<|J|$, then there exists $y \in J \backslash I$ such that $I \cup\{y\}$ is independent. 


\subsection{Examples.}

Example 3.2 (Linear matroids). Let $V$ be a vector space over a field $k$, and let $E$ be a finite subset of $V$. Define $\mathcal{I}$ to be the collection of linearly independent subsets of $E$. Then $\mathcal{I}$ satisfies (I1)-(I3) and therefore defines a matroid. Slightly more generally (because we allow repetitions), if $E=\{1, \ldots, m\}$ and $A$ is an $n \times m$ matrix with entries in $k$, a subset of $E$ is called independent iff the corresponding columns of $A$ are linearly independent over $k$. We denote this matroid by $M_{k}(A)$. A matroid of the form $M_{k}(A)$ for some $A$ is called representable over $k$.

By a recent theorem of Peter Nelson [23, asymptotically $100 \%$ of all matroids are not representable over any field.

Example 3.3 (Graphic matroids). Let $G$ be a finite graph, let $E$ be the set of edges of $G$, and let $\mathcal{I}$ be the collection of all subsets of $E$ which do not contain a cycle. Then $\mathcal{I}$ satisfies (I1)-(I3) and hence defines a matroid $M(G)$. The matroid $M(G)$ is regular, meaning that it is representable over every field $k$. By a theorem of Whitney, if $G$ is 3-connected (meaning that $G$ remains connected after removing any two vertices), then $M(G)$ determines the isomorphism class of $G$.

Example 3.4 (Uniform matroids). Let $E=\{1, \ldots, m\}$, and let $r$ be a positive integer. The uniform matroid $U_{r, m}$ is the matroid on $E$ whose independent sets are the subsets of $E$ of cardinality at most $r$. For each $r, m$ there exists $N=N(r, m)$ such that $U_{r, m}$ is representable over every field having at least $N(r, m)$ elements.

Example 3.5 (Fano matroid). Let $E=\mathbb{P}^{2}\left(\mathbf{F}_{2}\right)$ be the projective plane over the 2-element field; the seven elements of $E$ can be identified with the dots in Figure 2 ,

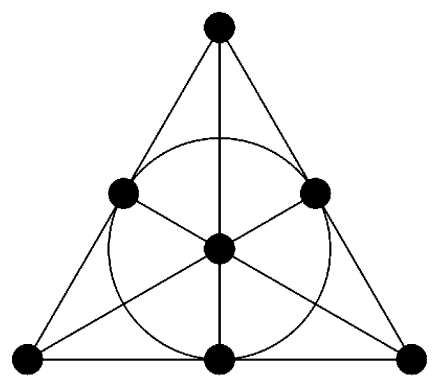

Figure 2. The Fano matroid

Define $\mathcal{I}$ to be the collection of subsets of $E$ of size at most 3 which are not one of the seven lines in $\mathbb{P}^{2}\left(\mathbf{F}_{2}\right)$ (depicted as six straight lines and a circle in Figure 2). Then $\mathcal{I}$ satisfies (I1)-(I3) and determines a matroid called the Fano matroid. This matroid is representable over $\mathbf{F}_{2}$ but not over any field of characteristic different from 2. In particular, the Fano matroid is not graphic.

Example 3.6 (Vamos matroid). Let $E$ be the eight vertices of the cuboid shown in Figure 3. Define $\mathcal{I}$ to be the collection of subsets of $E$ of size at most 4 which are not one of the five square faces in the figure. Then $\mathcal{I}$ satisfies (I1)-(I3) and determines a matroid called the Vamos matroid which is not representable over any field. 


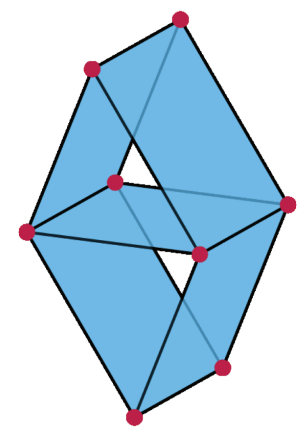

FiguRE 3. The Vamos matroid

3.3. Circuits, bases, and rank functions. A subset of $E$ which is not independent is called dependent. A minimal dependent set is called a circuit, and a maximal independent set is called a basis. As in linear algebra, all bases of $M$ have the same cardinality; this number is called the rank of the matroid $M$ and is denoted $r(M)$. More generally, if $A$ is a subset of $E$, we define the rank of $A$, denoted $r_{M}(A)$ or just $r(A)$, to be the maximal size of an independent subset of $A$.

One can give cryptomorphic axiomatizations of matroids in terms of circuits, bases, and rank functions. For the sake of brevity, we refer the interested reader to [25].

3.4. Duality. If $M=(E, \mathcal{I})$ is a matroid, let $\mathcal{I}^{*}$ be the collection of subsets $A \subseteq E$ such that $E \backslash A$ contains a basis $B$ for $M$. It turns out that $\mathcal{I}^{*}$ satisfies axioms (I1), (I2), and (I3), and thus $M^{*}=\left(E, \mathcal{I}^{*}\right)$ is a matroid, called the dual matroid of $M$.

If $M=M(G)$ is the matroid associated to a planar graph $G$, then $M^{*}$ is the matroid associated to the planar dual of $G$. A theorem of Whitney asserts, conversely, that if $G$ is a connected graph for which the dual matroid $M(G)^{*}$ is graphic, then $G$ is planar.

3.5. Deletion and contraction. Given a matroid $M$ on $E$ and $e \in E$, we write $M \backslash e$ for the matroid on $E \backslash\{e\}$ whose independent sets are the independent sets of $M$ not containing $e$.

We write $M / e$ for the matroid on $E \backslash\{e\}$ such that $I$ is independent in $M / e$ if and only if $I=J \backslash\{e\}$ with $J$ independent in $M$ and $e \in J$.

We call these operations on matroids deletion and contraction, respectively. Deletion and contraction are dual operations, in the sense that $(M \backslash e)^{*}=M^{*} / e$ and $(M / e)^{*}=M^{*} \backslash e$.

If $M$ is a graphic matroid, deletion and contraction correspond to the usual notions in graph theory.

3.6. Spans. We defined matroids in terms of independent sets, which abstract the notion of linear independence. We now focus on a different way to define/characterize matroids in terms of closure operators, which abstract the notion of span in linear algebra.

Let $2^{E}$ denote the power set of $E$. 
Definition 3.7 (Span axioms). A matroid $M$ is a finite set $E$ together with a function $\mathrm{cl}: 2^{E} \rightarrow 2^{E}$ such that for all $X, Y \subseteq E$ and $x, y \in E$ :

(S1) $X \subseteq \operatorname{cl}(X)$.

(S2) If $Y \subseteq X$, then $\operatorname{cl}(Y) \subseteq \operatorname{cl}(X)$.

(S3) $\operatorname{cl}(\operatorname{cl}(X))=\operatorname{cl}(X)$.

(S4) If $y \in \operatorname{cl}(X \cup\{x\})$ but $y \notin \operatorname{cl}(X)$, then $x \in \operatorname{cl}(X \cup\{y\})$.

For example, if $M$ is a linear matroid as in Example 3.2. then $\operatorname{cl}(X)$ is just the span of $X$ in $V$.

The exchange axiom (S4) captures our intuition of a "geometry" as a collection of incidence relations

$$
\{\text { point }\} \subset\{\text { line }\} \subset\{\text { plane }\} \subset \cdots .
$$

For example, if $L$ is a line in an $r$-dimensional projective space $\mathbb{P}_{k}^{r}$ over a field $k$ and $p, q \in \mathbb{P}_{k}^{r} \backslash L$, then $q$ lies in the span of $L \cup\{p\} \Longleftrightarrow p$ lies in the span of $L \cup\{q\}$ $\Longleftrightarrow p, q, L$ are coplanar.

The relation between Definitions 3.1 and 3.7 is simple to describe: given a matroid in the sense of Definition [3.1] we define $\operatorname{cl}(X)$ to be the set of all $x \in E$ such that $r(X \cup\{x\})=r(X)$. Conversely, given a matroid in the sense of Definition 3.7 we define a subset $I$ of $E$ to be independent if and only if $x \in I$ implies $x \notin \operatorname{cl}(I \backslash\{x\})$.

A subset $X$ of $E$ is said to span $M$ if $\operatorname{cl}(X)=E$. As in the familiar case of linear algebra, one can show in general that $X$ is a basis (i.e., a maximal independent set) if and only if $X$ is independent and spans $E$.

3.7. Flats. A subset $X$ of $E$ is called a flat (or a closed subset) if $X=\operatorname{cl}(X)$.

Example 3.8 (Linear matroids). Let $V$ be a vector space, and let $E$ be a finite subset of $V$. A subset $F$ of $E$ is a flat of the corresponding linear matroid if and only if there is no vector in $E \backslash F$ contained in the linear span of $F$.

Alternatively, let $M=M_{k}(A)$ be represented by an $r \times m$ matrix $A$ of rank $r$ with entries in $k$, and let $V \subseteq k^{m}$ be the row space of $A$. Let $E=\{1, \ldots, m\}$, and for $I \subseteq E$ let $L_{I}$ be the coordinate flat

$$
L_{I}=\left\{x=\left(x_{1}, \ldots, x_{m}\right) \in k^{m}: x_{i}=0 \text { for } i \in I\right\} .
$$

Then for $I \subseteq E$, we have $r_{M}(I)=\operatorname{dim}(V)-\operatorname{dim}\left(V \cap L_{I}\right)$, and $I$ is a flat of $M$ if and only if $V \cap L_{J} \subsetneq V \cap L_{I}$ for all $J \supsetneq I$. In particular, $V \cap L_{I}=V \cap L_{F}$, where $F$ is the smallest flat of $M$ containing $I$.

Example 3.9 (Graphic matroids). Let $G$ be a connected finite graph, and let $M(G)$ be the associated matroid. Then a subset $F$ of $E$ is a flat of $M(G)$ if and only if there is no edge in $E \backslash F$ whose endpoints are connected by a path in $F$.

Example 3.10 (Fano matroid). In the Fano matroid the flats are $\emptyset, E$, and each of the seven points and seven lines in Figure 2 .

Every maximal chain of flats of a matroid $M$ has the same length, which coincides with the rank of $M$.

One can give a cryptomorphic axiomatization of matroids in terms of flats. To state it, we say that a flat $F^{\prime}$ covers a flat $F$ if $F \subsetneq F^{\prime}$ and there are no intermediate flats between $F$ and $F^{\prime}$. 
Definition 3.11 (Flat axioms). A matroid $M$ is a finite set $E$ together with a collection of subsets of $E$, called flats, such that: 4

(F1) $E$ is a flat.

(F2) The intersection of two flats is a flat.

(F3) If $F$ is a flat and $\left\{F_{1}, F_{2}, \ldots, F_{k}\right\}$ is the set of flats that cover $F$, then $\left\{F_{1} \backslash F, F_{2} \backslash F, \ldots, F_{k} \backslash F\right\}$ partitions $E \backslash F$.

We have already seen how to define flats in terms of a closure operator. To go the other way, one defines the closure of a set $X$ to be the intersection of all flats containing $X$.

3.8. Simple matroids. A matroid $M$ is called simple if every dependent set has size at least 3. Equivalently, a matroid is simple if and only if it has no:

- loops (elements $e \in \operatorname{cl}(\emptyset)$ ); or

- parallel elements (elements $e, e^{\prime}$ with $\left.e^{\prime} \in \operatorname{cl}(e)\right) 5$

Every matroid $M$ has a canonical simplification $\hat{M}$ obtained by removing all loops and identifying parallel elements (with the obvious resulting notions of independence, closure, etc.). A simple matroid is also called a combinatorial geometry.

For future reference, we define a coloop of a matroid $M$ to be a loop of $M^{*}$. Equivalently, a loop is an element $e \in E$ which does not belong to any basis of $M$, and a coloop is an element $e \in E$ which belongs to every basis of $M$.

3.9. The Bergman fan of a matroid. Let $E=\{0,1, \ldots, n\}$, and let $M$ be a matroid on $E$. The Bergman fan of $M$ is a certain collection of cones in the $n$ dimensional Euclidean space $N_{\mathbb{R}}=\mathbb{R}^{E} / \mathbb{R}(1,1, \ldots, 1)$ which carries the same combinatorial information as $M$. Bergman fans show up naturally in the context of tropical geometry, where they are also known (in the trivially valued case) as tropical linear spaces.

For $S \subseteq E$, let $e_{S}=\sum_{i \in S} e_{i} \in N_{\mathbb{R}}$, where $e_{i}$ is the basis vector of $\mathbb{R}^{E}$ corresponding to $i$. Note that $e_{E}=0$ by the definition of $N_{\mathbb{R}}$. Let $F_{\bullet}=\left\{F_{1} \subsetneq F_{2} \subsetneq \cdots \subsetneq F_{k}\right\}$ be a $k$-step flag of nonempty proper flats of $M$. We define the corresponding cone $\sigma_{F_{\bullet}} \subseteq N_{\mathbb{R}}$ to be the nonnegative span of the $e_{F_{i}}$ for $i=1, \ldots, k$.

Definition 3.12. The Bergman fan $\Sigma_{M}$ of $M$ is the collection of cones $\sigma_{F_{\bullet}}$ as $F_{\bullet}$ ranges over all flags of nonempty proper flats of $M$.

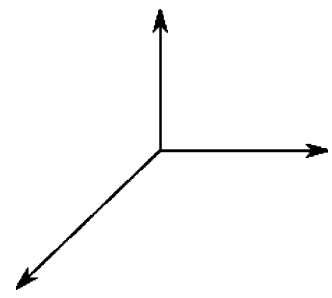

Figure 4. The Bergman fan of $U_{2,3}$

\footnotetext{
${ }^{4}$ For the geometric intuition behind axiom (F3) note that, given a line in $\mathbb{R}^{3}$, the planes which contain this line (minus the line itself) partition the remainder of $\mathbb{R}^{3}$.

"The terms "loop" and "parallel element" come from graph theory.
} 


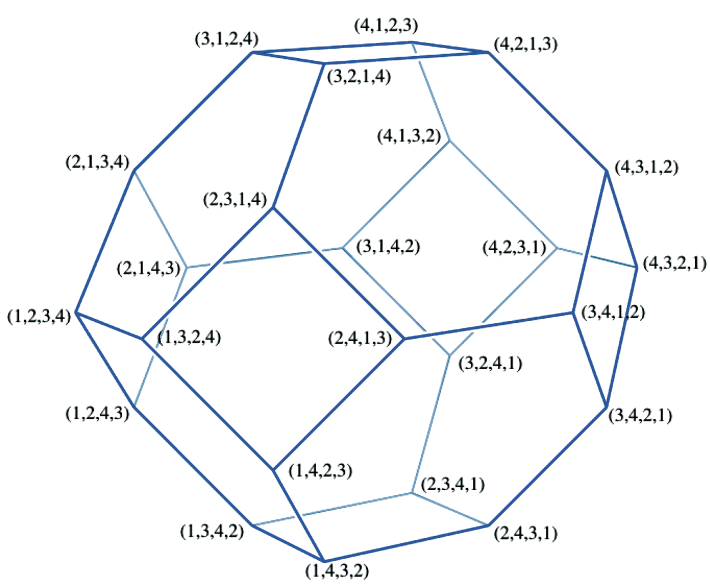

Figure 5. The three-dimensional permutohedron. Note that here we take $E=\{1,2,3,4\}$, instead of $\{0,1,2,3\}$ as in Example 3.14 , but this is immaterial since we work in $\mathbb{R}^{E} / \mathbb{R}(1,1, \ldots, 1)$.

Example 3.13. The Bergman fan of the uniform matroid $U_{2,3}$ has a zero-dimensional cone given by the origin in $\mathbb{R}^{2}$ and three one-dimensional cones given by rays from the origin in the directions of $\bar{e}_{1}=(1,0), \bar{e}_{2}=(0,1)$, and $\bar{e}_{3}=-\left(\bar{e}_{1}+\bar{e}_{2}\right)=$ $(-1,-1)$. This is the well-known tropical line in $\mathbb{R}^{2}$ with vertex at the origin; see Figure 4 .

Example 3.14. Let $U=U_{n+1, n+1}$ be the rank $n+1$ uniform matroid on $E=$ $\{0,1, \ldots, n\}$. Every subset of $E$ is a flat, so the top-dimensional cones of $\Sigma_{U}$ are the nonnegative spans of

$$
\left\{e_{i_{0}}, e_{i_{0}}+e_{i_{1}}, \ldots, e_{i_{0}}+e_{i_{1}}+\cdots+e_{i_{n-1}}\right\}
$$

for every permutation $i_{0}, \ldots, i_{n}$ of $0, \ldots, n$. The fan $\Sigma_{U}$ is the normal fan to the permutohedron $P_{n}$, which by definition is the convex hull of $\left(i_{0}, \ldots, i_{n}\right)$ over all permuations $i_{0}, \ldots, i_{n}$ of $0, \ldots, n$, viewed as a polytope in the dual vector space to $N_{\mathbb{R}}$; see Figure 5]. The fan $\Sigma_{U}$ plays a central and recurring role in [1]. For further information on some of the remarkable combinatorial and geometric properties of $P_{n}$, see 30.

The isomorphism class of matroid $M$ determines and is determined by its Bergman fan 6

\section{Geometric lattices and the Characteristic polynomial}

In this section we define the characteristic polynomial of a matroid $M$ in terms of the lattice of flats of $M$. Our primary references are [28] and [29, Chapters 7-8].

\footnotetext{
${ }^{6}$ One can in fact give a cryptomorphic characterization of matroids via their Bergman fans, using the flat axioms (F1)-(F3): a rational polyhedral fan $\Sigma$ in $N_{\mathbb{R}}$ is the Bergman fan of a matroid on $E$ if and only if it is balanced and has degree one as a tropical cycle [15].
} 
4.1. Geometric lattices. The set $\mathcal{L}(M)$ of flats of a matroid $M$ together with the inclusion relation forms a lattice, i.e., a partially ordered set in which every two elements $x, y$ have both a meet (greatest lower bound) $x \wedge y$ and a join (least upper bound) $x \vee y$. Indeed, if $X$ and $Y$ are flats, then we can define $X \wedge Y$ as the intersection of $X$ and $Y$ and $X \vee Y$ as the closure of the union of $X$ and $Y$.

Example 4.1. Flats of the uniform matroid $U_{n+1, n+1}$ can be identified with subsets of $\{0,1, \ldots, n\}$, and with this identification the lattice of flats of $U_{n+1, n+1}$ is the Boolean lattice $B_{n+1}$ consisting of subsets of $\{0,1, \ldots, n\}$ partially ordered by inclusion.

Example 4.2. Flats of the complete graph $K_{n}$ can be identified with partitions of $\{1, \ldots, n\}$, and with this identification the lattice of flats of the graphic matroid $M\left(K_{n}\right)$ is isomorphic to the partition lattice $\Pi_{n}$ consisting of partitions of $\{1, \ldots, n\}$ partially ordered by refinement.

If $L$ is a lattice and $x, y \in L$, we say that $y$ covers $x$ if $x<y$ and whenever $x \leq z \leq y$ we have either $z=x$ or $z=y$. A finite lattice has a minimal element $0_{L}$ and a maximal element $1_{L}$. An atom is an element which covers $0_{L}$.

The lattice of flats $L=\mathcal{L}(M)$ has the following properties:

(L1) $L$ is semimodular, i.e., if $x, y \in L$ both cover $x \wedge y$, then $x \vee y$ covers both $x$ and $y$.

(L2) $L$ is atomic, i.e., every $x \in L$ is a join of atoms.

A lattice satisfying (L1) and (L2) is called a geometric lattice. By a theorem of Garrett Birkhoff (the son of George), every geometric lattice is of the form $\mathcal{L}(M)$ for some matroid $M$. However, the matroid $M$ is not unique, because if $\hat{M}$ is the simplification of $M$, then $\mathcal{L}(M)=\mathcal{L}(\hat{M})$. Birkhoff proves that this is in fact the only ambiguity; i.e., the map $M \mapsto \mathcal{L}(M)$ gives a bijection between isomorphism classes of simple matroids and isomorphism classes of geometric lattices. Thus, at least up to simplification, (L1) and (L2) give another cryptomorphic characterization of matroids.

If $F$ is a flat of a matroid $M$, the maximal length $\ell$ of a chain $F_{0} \subset F_{1} \subset \cdots \subset$ $F_{\ell}=F$ of flats coincides with the $\operatorname{rank} r_{M}(F)$ of $F$. This allows us to define the rank function on $M$, restricted to the set of flats, purely in terms of the lattice $\mathcal{L}(M)$. We write $r_{L}$ for the corresponding function on an arbitrary geometric lattice $L$.

4.2. The Möbius function of a poset. There is a far-reaching combinatorial result known as the Möbius inversion formula which holds in an arbitrary finite poset $P$. It simultaneously generalizes, among other things, the inclusion-exclusion principle, the usual number-theoretic Möbius inversion formula, and the fundamental theorem of difference calculus.

There is a unique function $\mu_{P}: P \times P \rightarrow \mathbb{Z}$, called the Möbius function of $P$, satisfying $\mu_{P}(x, x)=1, \mu_{P}(x, y)=0$ if $x \not \leq y$, and

$$
\sum_{x \leq z \leq y} \mu_{P}(x, z)=0
$$

if $x<y$. Note that $\mu_{P}(x, y)=-1$ if $y$ covers $x$. 
The Möbius inversion formula states that if $f$ is a function from a finite poset $P$ to an abelian group $H$, and if we define $g(y)=\sum_{x \leq y} f(x)$ for all $y \in P$, then

$$
f(y)=\sum_{x \leq y} \mu_{P}(x, y) g(x) .
$$

If $P=L$ is a finite lattice, the Möbius function satisfies Weisner's theorem, which gives a "shortcut" for the recurrence defining $\mu$ : if $0_{L} \neq x \in L$, then

$$
\sum_{y \in L: x \vee y=1_{L}} \mu_{L}\left(0_{L}, y\right)=0 .
$$

If $L$ is moreover a geometric lattice, it is a theorem of Rota that the Möbius function of $L$ is nonzero and alternates in sign. More precisely, if $x \leq y$ in $L$, then

$$
(-1)^{r_{L}(y)-r_{L}(x)} \mu_{L}(x, y)>0 .
$$

4.3. The characteristic polynomial. The chromatic polynomial of a graph $G$ satisfies the deletion-contraction relation,

$$
\chi_{G}(t)=\chi_{G \backslash e}(t)-\chi_{G / e}(t) .
$$

Indeed, the equivalent formula $\chi_{G \backslash e}(t)=\chi_{G}(t)+\chi_{G / e}(t)$ just says that the proper colorings of $G \backslash e$ can be partitioned into those where the endpoints of $e$ are colored differently (giving a proper coloring of $G$ ) or the same (giving a proper coloring of $G / e$ ).

This formula is not only useful for calculating $\chi_{G}(t)$, it is also the simplest way to prove that $\chi_{G}(t)$ is a polynomial in $t$ (by induction on the number of edges). In addition, this formula for $\chi_{G}(t)$ suggests an extension to arbitrary matroids. This can be made to work, but it is not obvious that this recursive procedure is always well-defined. So it is more convenient to proceed as follows.

First, note that the chromatic polynomial of a graph $G$ is identically zero by definition if $G$ has a loop edge. So we will define $\chi_{M}(t)=0$ for any matroid with a loop. We may thus concentrate on loopless matroids. Note that a matroid $M$ is loopless if and only if $\emptyset$ is a flat of $M$.

Definition 4.3. Let $M$ be a loopless matroid with lattice of flats $L$. The characteristic polynomial of $M$ is

$$
\chi_{M}(t)=\sum_{F \in L} \mu_{L}(\emptyset, F) t^{r(M)-r(F)} .
$$

In particular, if $M$ is loopless, then $\chi_{M}(t)=\chi_{\hat{M}}(t)$, where $\hat{M}$ denotes the simplification of $M$.

The motivation behind (4.1) may be unclear to the reader at this point. In the representable case, at least, there is a motivic interpretation of (4.1) which some will find illuminating; see $\$ 4.6$.

There is also a (simpler looking but sometimes not as useful) expression for $\chi_{M}(t)$ in terms of a sum over all subsets of $E$, not just flats.

Proposition 4.4. If $M$ is any matroid,

$$
\chi_{M}(t)=\sum_{A \subseteq E}(-1)^{|A|} t^{r(M)-r(A)} .
$$


If $M_{1}, M_{2}$ are matroids on $E_{1}$ and $E_{2}$, respectively, and $E_{1} \cap E_{2}=\emptyset$, we define the direct sum $M_{1} \oplus M_{2}$ to be the matroid on $E_{1} \cup E_{2}$ whose flats are all sets of the form $F_{1} \cup F_{2}$ where $F_{i}$ is a flat of $M_{i}$ for $i=1,2$. The following result gives an important characterization of the characteristic polynomial.

Theorem 4.5. Let $M$ be a matroid.

$(\chi 1)$ If $e$ is neither a loop nor a coloop of $M$, then $\chi_{M}(t)=\chi_{M \backslash e}(t)-\chi_{M / e}(t)$.

$(\chi 2)$ If $M=M_{1} \oplus M_{2}$, then $\chi_{M}(t)=\chi_{M_{1}}(t) \chi_{M_{2}}(t)$.

$(\chi 3)$ If $M$ contains a loop, then $\chi_{M}(t)=0$, and if $M$ consists of a single coloop, then $\chi_{M}(t)=t-1$.

Furthermore, the characteristic polynomial is the unique function from matroids to integer polynomials satisfying $(\chi 1)-(\chi 3)$.

In particular, it follows from Theorem 4.5 that if $G$ is a graph, then the chromatic polynomial $\chi_{G}(t)$ of $G$ satisfies $\chi_{G}(t)=t^{c(G)} \chi_{M(G)}(t)$, where $c(G)$ is the number of connected components of $G$. (The extra factor of $t$ when $G$ is connected comes from the fact that the graph with two vertices and one edge has chromatic polynomial $t(t-1)$, whereas the corresponding matroid, which consists of a single coloop, has characteristic polynomial $t-1$. Note that since no graph can be 0 -colored, $\chi_{G}(0)=0$ for every graph $G$ and hence the chromatic polynomial is always divisible by $t$.)

The characteristic polynomial of $M$ is monic of degree $r=r(M)$, so we can write

$$
\chi_{M}(t)=w_{0}(M) t^{r}+w_{1}(M) t^{r-1}+\cdots+w_{r}(M)
$$

with $w_{0}(M)=1$ and $w_{k}(M) \in \mathbb{Z}$. By Rota's theorem, the coefficients of $\chi_{M}(t)$ alternate in sign, i.e.,

$$
w_{k}(M)^{+}:=\left|w_{k}(M)\right|=(-1)^{k} w_{k}(M) .
$$

The numbers $w_{k}(M)$ (resp. $\left.w_{k}^{+}(M)\right)$ are called the Whitney numbers of the first kind (resp. unsigned Whitney numbers of the first kind) for $M$. The recent work [1] of Adiprasito, Huh, and Katz establishes:

Theorem 4.6. For any matroid $M$, the unsigned Whitney numbers of the first kind $w_{k}^{+}(M)$ form a log-concave sequence.

Note that it is enough to prove the theorem for simple matroids, i.e., combinatorial geometries, since the characteristic polynomial of a loopless matroid equals that of its simplification.

Actually, Adiprasito, Huh, and Katz study the so-called reduced characteristic polynomial of $M$. If $|E| \geq 1$, then $\chi_{M}(1)=0$; e.g., if $G$ is a graph with at least one edge, then $G$ has no proper one-coloring! Thus we may write $\chi_{M}(t)=(t-1) \bar{\chi}_{M}(t)$ with $\bar{\chi}_{M}(t) \in \mathbb{Z}[t]$. The reduced characteristic polynomial $\bar{\chi}_{M}(t)$ is the projective analogue of $\chi_{M}(t)$ (cf. $\$ 4.6$ below). It is an elementary fact that log-concavity of the (absolute values of the) coefficients of $\bar{\chi}_{M}(t)$ implies log-concavity for $\chi_{M}(t)$. So in order to prove Theorem 4.6. one can replace the $w_{k}^{+}(M)$ by their projective analogues $m_{k}(M)$.

4.4. Tutte-Grothendieck invariants. Our primary reference for this section and 4.6 is 20 .

One can generalize the characteristic polynomial of a matroid by relaxing the condition that it vanishes on matroids containing loops. 
The Tutte polynomial of a matroid $M$ on $E$ is the two-variable polynomial

$$
T_{M}(x, y)=\sum_{A \subseteq E}(x-1)^{r(M)-r(A)}(y-1)^{|A|-r(A)} .
$$

By (4.2), we have $\chi_{M}(t)=(-1)^{r(M)} T_{M}(1-t, 0)$.

To put Theorem 4.5 into perspective, we define the Tutte-Grothendieck ring of matroids to be the commutative ring $K_{0}$ (Mat) defined as the free abelian group on isomorphism classes of matroids, together with multiplication given by the direct sum of matroids, modulo the relations that if $e$ is neither a loop nor a coloop of $M$, then $[M]=[M \backslash e]+[M / e]$.

If $R$ is a commutative ring, an $R$-valued Tutte-Grothendieck invariant is a homomorphism from $K_{0}$ (Mat) to $R$. The following result, due to Crapo and Brylawski, asserts that the Tutte polynomial is the universal Tutte-Grothendieck invariant:

Theorem 4.7. (1) The Tutte polynomial is the unique Tutte-Grothendieck invariant $T: K_{0}$ (Mat) $\rightarrow \mathbb{Z}[x, y]$ satisfying $T$ (coloop) $=x$ and $T($ loop $)=y$.

(2) More generally, if $\phi: K_{0}$ (Mat) $\rightarrow R$ is any Tutte-Grothendieck invariant, then $\phi=\phi_{0} \circ T$, where $\phi_{0}: \mathbb{Z}[x, y] \rightarrow R$ is the unique ring homomorphism sending $x$ to $\phi$ (coloop) and $y$ to $\phi$ (loop).

Similarly, the characteristic polynomial is the universal Tutte-Grothendieck invariant for combinatorial geometries. More precisely, if $\phi$ is any Tutte-Grothendieck invariant such that $\phi(M)=\phi(\hat{M})$ for every loopless matroid $M$, then

$$
\phi(M)=(-1)^{r(M)} \chi_{M}(1-\phi(\text { coloop })) .
$$

The Tutte polynomial has a number of remarkable properties. For example, one has the following compatibility with matroid duality:

$$
T_{M}(x, y)=T_{M^{*}}(y, x) .
$$

4.5. The rank polynomial. Let $M$ be a simple matroid with lattice of flats $L$. The rank polynomial of $M$ is

$$
\rho_{M}(t)=\sum_{F \in L} t^{r(M)-r(F)}=W_{0}(M) t^{r}+W_{1}(M) t^{r-1}+\cdots+W_{r}(M) .
$$

The coefficients $W_{k}(M)$ of $\rho_{M}(t)$ are strictly positive, and are called the Whitney numbers of the second kind. Concretely, $W_{k}(M)$ is the number of flats in $M$ of rank $k$. Comparing with (4.1), we see that the coefficients of $\chi_{M}(t)$ and $\rho_{M}(t)$ are related by

$$
\begin{aligned}
w_{k}(M) & =\sum_{F \in L: r(F)=k} \mu_{L}(\emptyset, F), \\
W_{k}(M) & =\sum_{F \in L: r(F)=k} 1 .
\end{aligned}
$$

For the matroid $M_{n}:=M\left(K_{n}\right)$ associated to the complete graph $K_{n}, w_{k}\left(M_{n}\right)=$ $s(n, k)$ and $W_{k}\left(M_{n}\right)=S(n, k)$ are the Stirling numbers of the first and second kind, respectively (hence the name for the Whitney numbers).

It is conjectured that the Whitney numbers of the second kind form a logconcave, and hence unimodal, sequence for every simple matroid $M$. This, however, remains an open problem. 
It is a recent theorem of Huh and Wang [19] that if $M$ is a rank $r$ matroid which is representable over some field, then $W_{1} \leq W_{2} \leq \cdots \leq W_{\lfloor r / 2\rfloor}$ and $W_{k} \leq W_{r-k}$ for every $k \leq r / 2$; see $\$ 5.10$ below.

4.6. Motivic interpretation of the characteristic polynomial. Let $k$ be a field. The Grothendieck ring of $k$-varieties is the commutative ring $K_{0}\left(\operatorname{Var}_{k}\right)$ defined as the free abelian group on isomorphism classes of $k$-varieties, together with multiplication given by the product of varieties, modulo the scissors congruence relations that whenever $Z \subset X$ is a closed $k$-subvariety, we have $[X]=[X \backslash Z]+[Z]$.

When $k=\mathbb{C}$ or $k=\mathbb{F}_{q}$ is a finite field, there is a canonical ring homomorphism 7 $e: K_{0}\left(\operatorname{Var}_{k}\right) \rightarrow \mathbb{Z}[t]$ with the property that $e\left(\mathbb{A}_{k}^{1}\right)=t$.

Let $A$ be an $r \times m$ matrix with entries in $k$ representing a rank $r$ matroid $M$ with lattice of flats $L$. Let $V \subset k^{m}$ be the row space of $A$. With the notation of Example 3.8 the Möbius inversion formula shows that in the ring $K_{0}\left(\operatorname{Var}_{k}\right)$, we have the motivic identity

$$
\left[V \cap\left(k^{\times}\right)^{m}\right]=\sum_{F \in L} \mu_{L}\left(0_{L}, F\right)\left[V \cap L_{F}\right] .
$$

(For example, if $V$ is a generic subspace of $k^{m}$, then by inclusion-exclusion we have

$$
\left[V \cap\left(k^{\times}\right)^{m}\right]=\left[V \cap L_{\emptyset}\right]-\sum_{i}\left[V \cap L_{i}\right]+\sum_{|I|=2}\left[V \cap L_{I}\right]-\cdots,
$$

but in general there are subspace relations between the various $V \cap L_{I}$ governed by the combinatorics of the underlying matroid.)

The identity (4.3), which is strongly reminiscent of (4.1), can be used to establish Theorem 4.5 in the representable case. Since $e\left(V \cap L_{F}\right)=t^{r-r(F)}$, it also explains the theorem of Orlik and Solomon 24 that for $k=\mathbb{C}$ the Hodge polynomial of $V \cap\left(\mathbb{C}^{*}\right)^{m}$ is $\chi_{M}(t)$, as well as the theorem of Athanasiadis [3] that for $k=\mathbb{F}_{q}$ with $q$ sufficiently large, we have

$$
\left|V \cap\left(\mathbb{F}_{q}^{\times}\right)^{m}\right|=\chi_{M}(q) .
$$

We mentioned in section 4.3 that the reduced characteristic polynomial $\bar{\chi}_{M}(t)$ is the projective analogue of $\chi_{M}(t)$. A concrete way to interpret this statement in the representable case is that since $k^{\times}$, which satisfies $e\left(k^{\times}\right)=t-1$, acts freely on $V \cap\left(k^{\times}\right)^{m}$, we have

$$
e\left(\mathbb{P}\left(V \cap\left(k^{\times}\right)^{m}\right)\right)=e\left(V \cap\left(k^{\times}\right)^{m}\right) / e\left(k^{\times}\right)=\chi_{M}(t) /(t-1)=\bar{\chi}_{M}(t) .
$$

\section{Overview of the proof of the Rota-Welsh conjecture}

We briefly outline the strategy used by Adiprasito, Huh, and Katz in their proof of the Rota-Welsh conjecture. (See [2] for another survey of the proof.) The first step is to define a Chow ring $A^{*}(M)$ associated to an arbitrary loopless matroid $M$. The definition of this ring is motivated by work of Feichtner and Yuzvinsky [14], who noted that when $M$ is realizable over $\mathbf{C}$, the $\operatorname{ring} A^{*}(M)$ coincides with the usual Chow ring of the de Concini-Procesi wonderful compactification $Y_{M}$ of the hyperplane arrangement complement associated to $M$ 11, 12, 8 (Although the

\footnotetext{
${ }^{7}$ This homomorphism may be defined when $k=\mathbb{C}$ as the compactly supported $\chi_{y}$-genus from mixed Hodge theory, and when $k=\mathbb{F}_{q}$ as the compactly supported $\chi_{y}$-genus in $\ell$-adic cohomology.

${ }^{8}$ Technically speaking, there are different wonderful compactification in the work of de Concini and Procesi; the one relevant for [1] corresponds to the finest building set.
} 
definition of $A^{*}(M)$ is purely combinatorial and does not require any notions from algebraic geometry, it would presumably be rather hard to motivate the following definition without knowing something about the relevant geometric background.) Note that $Y_{M}$ is a smooth projective variety of dimension $d:=r-1$, where $r$ is the rank of $M$.

5.1. The Chow ring of a matroid. Let $M$ be a loopless matroid, and let $\mathcal{F}^{\prime}=$ $\mathcal{F} \backslash\{\emptyset, E\}$ be the poset of nonempty proper flats of $M$. The graded ring $A^{*}(M)$ is defined as the quotient of the polynomial ring $S_{M}=\mathbf{Z}\left[x_{F}\right]_{F \in \mathcal{F}^{\prime}}$ by the following two kinds of relations:

(CH1) For every $a, b \in E$, the sum of the $x_{F}$ for all $F$ containing $a$ equals the sum of the $x_{F}$ for all $F$ containing $b$.

(CH2) $x_{F} x_{F^{\prime}}=0$ whenever $F$ and $F^{\prime}$ are incomparable in the poset $\mathcal{F}^{\prime}$.

The generators $x_{F}$ are viewed as having degree one. There is an isomorphism 9 $\operatorname{deg}: A^{d}(M) \rightarrow \mathbf{Z}$ determined uniquely by the property that $\operatorname{deg}\left(x_{F_{1}} x_{F_{2}} \cdots x_{F_{d}}\right)=$ 1 whenever $F_{1} \subsetneq F_{2} \subsetneq \cdots \subsetneq F_{d}$ is a maximal flag in $\mathcal{F}^{\prime}$.

It may be helpful to note that $A^{*}(M)$ can be naturally identified with equivalence classes of piecewise polynomial functions on the Bergman fan $\Sigma_{M}$. The fact that there is a unique homomorphism $\operatorname{deg}: A^{d}(M) \rightarrow \mathbf{Z}$ as above means, in the language of tropical geometry, that there is a unique (up to scalar multiple) set of integer weights on the top-dimensional cones of $\Sigma_{M}$ which make it a balanced polyhedral complex.

5.2. Connection to Hodge theory. If $M$ is realizable, one can use the so-called Hodge-Riemann relations from algebraic geometry, applied to the smooth projective algebraic variety $Y_{M}$ whose Chow ring is $A^{*}(M)$, to prove the Rota-Welsh logconcavity conjecture for $M$. This is (in retrospect, anyway) the basic idea in the earlier paper of Huh and Katz, about which we will say more in section 5.8 below.

We now quote from the introduction to [1]:

While the Chow ring of $M$ could be defined for arbitrary $M$, it was unclear how to formulate and prove the Hodge-Riemann relations... We are nearing a difficult chasm, as there is no reason to expect a working Hodge theory beyond the case of realizable matroids. Nevertheless, there was some evidence on the existence of such a theory for arbitrary matroids.

What the authors of [1] do is to formulate a purely combinatorial analogue of the hard Lefschetz theorem and Hodge-Riemann relations and prove them for the ring $A^{*}(M)_{\mathbf{R}}:=A^{*}(M) \otimes \mathbf{R}$ in a purely combinatorial way, making no use of algebraic geometry. The idea is that although the ring $A^{*}(M)_{\mathbf{R}}$ is not actually the cohomology ring of a smooth projective variety, from a Hodge-theoretic point of view it behaves as if it were.

5.3. Ample classes, hard Lefschetz, and Hodge-Riemann. In order to formulate precisely the main theorem of [1, we need a combinatorial analogue of hyperplane classes, or more generally of ample divisors. The connection goes through strictly submodular functions.

A function $c: 2^{E} \rightarrow \mathbf{R}_{\geq 0}$ is called strictly submodular if $c(E)=c(\emptyset)=0$ and $c(A \cup B)+c(A \cap B)<c(A)+c(B)$ whenever $A, B$ are incomparable subsets of

\footnotetext{
${ }^{9}$ The isomorphism deg should not be confused with the grading on the $\operatorname{ring} A^{*}(M)$ : these are two different usages of the term "degree".
} 
$E$. Strictly submodular functions exist, and each such $c$ gives rise to an element $\ell(c)=\sum_{F \in \mathcal{F}^{\prime}} c(F) x_{F} \in A^{1}(M)_{\mathbb{R}}$. The convex cone of all $\ell(c) \in A^{1}(M)_{\mathbb{R}}$ associated to strictly submodular classes is called the ample cone 10 and elements of the form $\ell(c)$ are called ample classes in $A^{1}(M)_{\mathbb{R}}$.

Ample classes in $A^{1}(M)_{\mathbb{R}}$ correspond in a natural way to strictly convex piecewise-linear functions on the Bergman fan $\Sigma_{M}$ (cf. \$3.9).

The main theorem of [1] is the following:

Theorem 5.1 (Adiprasito, Huh, and Katz, 2015). Let $M$ be a matroid of rank $r=d+1$, let $\ell \in A^{1}(M)_{\mathbf{R}}$ be ample, and let $0 \leq k \leq \frac{d}{2}$. Then:

(1) Poincaré duality: The natural multiplication map gives a perfect pairing $A^{k}(M) \times A^{d-k}(M) \rightarrow A^{d}(M) \cong \mathbf{Z}$.

(2) Hard Lefschetz theorem: Multiplication by $\ell^{d-2 k}$ determines an isomorphism $L_{\ell}^{k}: A^{k}(M)_{\mathbf{R}} \rightarrow A^{d-k}(M)_{\mathbf{R}}$.

(3) Hodge-Riemann relations: The natural bilinear form

$$
Q_{\ell}^{k}: A^{k}(M)_{\mathbf{R}} \times A^{k}(M)_{\mathbf{R}} \rightarrow \mathbf{R}
$$

defined by $Q_{\ell}^{k}(a, b)=(-1)^{k} a \cdot L_{\ell}^{k} b$ is positive definite on the kernel of $\ell \cdot L_{\ell}^{k}$ (the so-called primitive classes).

This is all in very close analogy with analogous results in classical Hodge theory.

5.4. Combinatorial Hodge theory implies the Rota-Welsh conjecture. To see why Theorem 5.1 implies the Rota-Welsh conjecture, fix $e \in E=\{0, \ldots, n\}$. Let $\alpha(e) \in S_{M}$ be the sum of $x_{F}$ over all $F$ containing $e$, and let $\beta(e) \in S_{M}$ be the sum of $x_{F}$ over all $F$ not containing $e$. The images of $\alpha(e)$ and $\beta(e)$ in $A^{1}(M)$ do not depend on $e$, and are denoted by $\alpha$ and $\beta$, respectively.

Theorem 5.2. Let $\bar{\chi}_{M}(t):=\chi_{M}(t) /(t-1)$ be the reduced characteristic polynomial of $M$, and write $\bar{\chi}_{M}(t)=m_{0} t^{d}-m_{1} t^{d-1}+\cdots+(-1)^{d} m_{d}$. Then $m_{k}=\operatorname{deg}\left(\alpha^{d-k} \cdot \beta^{k}\right)$ for all $k=0, \ldots, d$.

The proof of this result is based on the following positive combinatorial formula for $m_{k}$ due originally to Björner [5,6]. (It can also be deduced as a straightforward consequence of Weisner's theorem.)

A $k$-step flag $F_{1} \subsetneq F_{2} \subsetneq \cdots \subsetneq F_{k}$ in $\mathcal{F}^{\prime}$ is said to be initial if $r_{M}\left(F_{i}\right)=i$ for all $i$, and descending if

$$
\min \left(F_{1}\right)>\min \left(F_{2}\right)>\cdots>\min \left(F_{k}\right)>0,
$$

where for $F \subseteq\{0,1, \ldots, n\}$ we set $\min (F)=\min \{i: i \in F\}$.

Proposition 5.3. $m_{k}$ is the number of initial, descending $k$-step flags in $\mathcal{F}^{\prime}$.

Although $\alpha$ and $\beta$ are not ample, one may view them as a limit of ample classes (i.e., they belong to the nef cone). This observation, together with the HodgeRiemann relations for $A^{0}(M)$ and $A^{1}(M)$ and Theorem 5.2, allows one to deduce the Rota-Welsh conjecture in a formal way.

\footnotetext{
${ }^{10}$ Actually, the ample cone in [1] is a priori larger than what we've just defined, but this subtlety can be ignored for the present purposes.
} 
5.5. Log-concavity of $f$-vectors of matroids. The Rota-Welsh conjecture implies a conjecture of Mason and Welsh on $f$-vectors of matroids.

Corollary 5.4 (Mason-Welsh conjecture). Let $M$ be a matroid on $E$, and let $f_{k}(M)$ be the number of independent subsets of $E$ with cardinality $k$. Then the sequence $f_{k}(M)$ is log-concave and hence unimodal.

To deduce Corollary 5.4 from the results of [1, one proceeds by showing that the signed $f$-polynomial

$$
f_{0}(M) t^{r}-f_{1}(M) t^{r-1}+\cdots+(-1)^{r} f_{r}(M)
$$

of the rank $r$ matroid $M$ coincides with the reduced characteristic polynomial of an auxiliary rank $r+1$ matroid $M^{\prime}$ constructed from $M$, the so-called free co-extension of $M$ This identity was originally proved by Brylawski 7] and subsequently rediscovered by Lenz [21].

5.6. High-level overview of the strategy for proving Theorem [5.1, The main work in [1] is of course establishing Poincaré duality and especially the hard Lefschetz theorem and Hodge-Riemann relations for $M$. From a high-level point of view, the proof is reminiscent of Peter McMullen's strategy in [22], where he reduces the so-called $g$-conjecture 12 for arbitrary simple polytopes to the case of simplices using the flip connectivity of simple polytopes of given dimension.

A key observation in [1, motivated in part by McMullen's work, is that for any two matroids $M$ and $M^{\prime}$ of the same rank on the same ground set $E$, there is a diagram

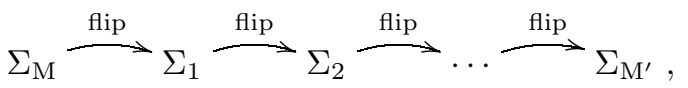

where each matroidal flip 13 preserves the validity of the hard Lefschetz theorem and Hodge-Riemann relations 14 Using this, one reduces Theorem 5.1 to the HodgeRiemann relations for projective space, which admit a straightforward (and purely combinatorial) proof.

The inductive approach to the hard Lefschetz theorem and the Hodge-Riemann relations in [1] is modeled on the observation that any facet of a permutohedron is the product of two smaller permutohedrons.

5.7. Remarks on Chow equivalence. The Chow ring $A^{*}(M)$ of a rank $d+1$ matroid $M$ on $\{0, \ldots, n\}$ coincides with the Chow ring of the smooth but noncomplete toric variety $X\left(\Sigma_{M}\right)$ associated to the Bergman fan of $M$. One of the subtleties here, and one of the remarkable aspects of the results in [1], is that

\footnotetext{
${ }^{11}$ To define the free co-extension, let $e$ be an auxiliary element not in $E$, and let $E^{\prime}=E \cup$ $\{e\}$. The free extension of $M$ by $e$ is the matroid $M+e$ on $E^{\prime}$ whose independent sets are the independent sets of $M$ together with all sets of the form $I \cup\{e\}$ with $I$ an independent set of $M$ of cardinality at most $r-1$. The free co-extension of $M$ by $e$ is the matroid $M \times e$ on $E^{\prime}$ given by $M \times e=\left(M^{*}+e\right)^{*}$.

${ }^{12}$ For an overview of the $g$-conjecture and applications of Hodge theory to the enumerative geometry of polytopes, see, e.g., Richard Stanley's article [27.

${ }^{13}$ A word of caution about the terminology: although these operations are called "flips" in [1, they are not analogous to flips in the sense of birational geometry but rather to blowups and blowdowns.

${ }^{14} \mathrm{~A}$ subtlety is that the intermediate objects $\Sigma_{i}$ are balanced weighted rational polyhedral fans but not necessarily tropical linear spaces associated to some matroid. So one leaves the world of matroids in the course of the proof, unlike with McMullen's case of polytopes.
} 
although the $n$-dimensional toric variety $X\left(\Sigma_{M}\right)$ is not complete, its Chow ring "behaves like" the Chow ring of a $d$-dimensional smooth projective variety.

When $M$ is representable over a field $k$, there is a good reason for this: one can construct a map from a smooth projective variety $Y$ of dimension $d$ to $X\left(\Sigma_{M}\right)$ which induces (via pullback) an isomorphism of Chow rings

$$
A^{*}\left(X\left(\Sigma_{M}\right)\right) \stackrel{\sim}{\longrightarrow} A^{*}(Y) .
$$

(We call such an isomorphism a Chow equivalence.)

For example, if $M=U_{2,3}$ is the uniform matroid represented over $\mathbb{C}$ by a line $\ell \subset \mathbb{P}^{2}$ in general position, its Bergman fan $\Sigma_{M}$ is a tropical line in $\mathbb{R}^{2}$ (cf. Example 3.13) and the corresponding toric variety $X\left(\Sigma_{M}\right)$ is isomorphic to $\mathbb{P}^{2} \backslash\{0,1, \infty\}$. Pullback along the inclusion map $\mathbb{P}^{1} \cong \ell \hookrightarrow \mathbb{P}^{2} \backslash\{0,1, \infty\}$ induces a Chow equivalence between $\mathbb{P}^{2} \backslash\{0,1, \infty\}$ and $\mathbb{P}^{1}$. (However, the induced map $H^{*}\left(\mathbb{P}^{2} \backslash\{0,1, \infty\}, \mathbb{C}\right) \rightarrow H^{*}\left(\mathbb{P}^{1}, \mathbb{C}\right)$ on singular cohomology rings is far from being an isomorphism.)

When $M$ is not realizable, however, there is provably no such Chow equivalence between $A^{*}(M)$ and the Chow ring of a smooth projective variety $Y$ mapping to $X\left(\Sigma_{M}\right)$ [1, Theorem 5.12].

The construction of $Y$ in the realizable case follows from the theory of de ConciniProcesi wonderful compactifications. One takes the toric variety $X\left(\Sigma_{U}\right)$ associated to the $n$-dimensional permutohedron $P_{n}$ (cf. 93.9 ) - the so-called permutohedral variety 15 and views the Bergman fan $\Sigma_{M}$ of the realizable rank $d+1$ matroid $M$ as a $d$-dimensional subfan of the normal fan $\Sigma_{U}$ to $P_{n}$, which is a complete $n$-dimensional fan in $\mathbb{R}^{n}$. This induces an open immersion of toric varieties $X\left(\Sigma_{M}\right) \subset X\left(\Sigma_{U}\right)$, and the wonderful compactification $Y$ of the hyperplane arrangement complement realizing $M$, which is naturally a closed subvariety of $X\left(\Sigma_{U}\right)$, belongs to the open subset $X\left(\Sigma_{M}\right)$. The induced inclusion map $Y \hookrightarrow X\left(\Sigma_{M}\right)$ realizes the desired Chow equivalence.

In this case, the linear relations $(\mathrm{CH} 1)$ come from linear equivalence on the ambient permutohedral toric variety $X\left(\Sigma_{U}\right)$, pulled back along the open immersion $X\left(\Sigma_{M}\right) \hookrightarrow X\left(\Sigma_{U}\right)$, and the quadratic relations (CH2) come from the fact that if $F$ and $F^{\prime}$ are incomparable flats, then the corresponding divisors are disjoint in $X\left(\Sigma_{U}\right)$.

5.8. Proof of log-concavity in the realizable case d'après Huh and Katz. The geometric motivation for several parts of the proof of the Rota-Welsh conjecture comes from the proof of the representable case given in 18, and it is intimately connected with the geometry of the permutohedral variety. (We remind the reader, however, that asymptotically $100 \%$ of all matroids are not representable over any field [23].) We briefly sketch the argument from [18.

The $n$-dimensional permutohedral variety $X\left(\Sigma_{U}\right)$ is a smooth projective variety which can be considered as an iterated blowup of $\mathbb{P}^{n}$. After fixing homogenous coordinates on $\mathbb{P}^{n}$, we get a number of distinguished linear subspaces of $\mathbb{P}^{n}$, for example the $n+1$ points having all but one coordinate equal to zero. We also get the coordinate lines between any two of those points, and in general we can consider all linear subspaces of the form $\bigcap_{i \in I} H_{i}$ where $H_{i}$ is the $i$ th coordinate hyperplane and $I \subset E:=\{0,1, \ldots, n\}$. The permutohedral variety $X\left(\Sigma_{U}\right)$ can be constructed by first blowing up the $n+1$ coordinate points, then blowing up the

\footnotetext{
${ }^{15}$ The permutohedral variety is an example of a Losev-Manin moduli space.
} 
proper transforms of the coordinate lines, then blowing up the proper transforms of the coordinate planes, and so on. In particular, this procedure determines a distinguished morphism $\pi_{1}: X\left(\Sigma_{U}\right) \rightarrow \mathbb{P}^{n}$ which is a proper modification of $\mathbb{P}^{n}$.

There is another distinguished morphism $\pi_{2}: X\left(\Sigma_{U}\right) \rightarrow \mathbb{P}^{n}$ which can be obtained by composing $\pi_{1}$ with the standard Cremona transform Crem : $\mathbb{P}^{n} \rightarrow-\mathbb{P}^{n}$ given in homogeneous coordinates by $\left(x_{0}: \cdots: x_{n}\right) \mapsto\left(x_{0}^{-1}: \cdots: x_{n}^{-1}\right)$. Although Crem is only a rational map on $\mathbb{P}^{n}$, it extends to an automorphism of $X\left(\Sigma_{U}\right)$, i.e., there is a morphism $\widetilde{\mathrm{Crem}}: X\left(\Sigma_{U}\right) \rightarrow X\left(\Sigma_{U}\right)$ such that $\pi_{1} \circ \widetilde{\mathrm{Crem}}=$ Crem $\circ \pi_{1}$ as rational maps $X\left(\Sigma_{U}\right) \rightarrow \mathbb{P}^{n}$. In other words, $\widetilde{\text { Crem }}: X\left(\Sigma_{U}\right) \rightarrow X\left(\Sigma_{U}\right)$ resolves the indeterminacy locus of Crem. We set $\pi_{2}=\pi_{1} \circ \widetilde{\text { Crem. }}$.

A rank $d+1$ loopless matroid $M$ on $E$ which is representable over $k$ corresponds to a $(d+1)$-dimensional subspace $V$ of $k^{n+1}$ which is not contained in any hyperplane. Let $\mathbb{P}(V) \subset \mathbb{P}^{n}$ be the projectivization of $V$. Like $X\left(\Sigma_{U}\right)$ itself, the proper transform $\widetilde{\mathbb{P}(V)}$ of $\mathbb{P}(V)$ in $X\left(\Sigma_{U}\right)$ can be constructed as an iterated blowup, in this case a blowup of $\mathbb{P}(V)$ at its intersections with the various coordinate spaces of $\mathbb{P}^{n}$. In fact, $\widetilde{\mathbb{P}(V)}$ coincides with the de Concini-Procesi wonderful compactification $Y$ mentioned above. The homology class of $\widetilde{\mathbb{P}(V)}$ in the permutohedral variety depends only on the matroid $M$, and not on the particular choice of the subspace $V$. We denote by $p_{1}, p_{2}$ the restrictions to $\widetilde{\mathbb{P}(V)}$ of $\pi_{1}, \pi_{2}$, respectively.

The key fact from $[18$ linking $\widetilde{\mathbb{P}(V)}$ and the ambient permutohedral variety to the Rota-Welsh conjecture is the following (compare with Theorem [5.2):

Theorem 5.5. Let $H$ be the class of a hyperplane in $\operatorname{Pic}\left(\mathbb{P}^{n}\right)$, let $\alpha=p_{1}^{-1}(H)$, and let $\beta=p_{2}^{-1}(H)$. Then:

(1) The class of $\left(p_{1} \times p_{2}\right)(\widetilde{\mathbb{P}(V)})$ in the Chow ring of $\mathbb{P}^{n} \times \mathbb{P}^{n}$ is

$$
m_{0}\left[\mathbb{P}^{d} \times \mathbb{P}^{0}\right]+m_{1}\left[\mathbb{P}^{d-1} \times \mathbb{P}^{1}\right]+\cdots+m_{r}\left[\mathbb{P}^{0} \times \mathbb{P}^{d}\right] .
$$

(2) The kth coefficient $m_{k}$ of the reduced characteristic polynomial $\bar{\chi}_{M}(t)$ is equal to $\operatorname{deg}\left(\alpha^{d-k} \beta^{k}\right)$.

The Rota-Welsh conjecture for representable matroids follows immediately from Theorem $5.5(2)$ and the Khovanskii-Teissier inequality, which says that if $X$ is a smooth projective variety of dimension $d$ and $\alpha, \beta$ are nef divisors on $X$, then $\operatorname{deg}\left(\alpha^{d-k} \beta^{k}\right)$ is a log-concave sequence.

5.9. The Kähler package. The proof of the Khovanskii-Teissier inequality uses Kleiman's criterion to reduce to the case where $\alpha, \beta$ are ample, then uses the Kleiman-Bertini theorem to reduce to the case of surfaces, in which case the desired inequality is precisely the classical Hodge index theorem. The Hodge index theorem itself is a very special case of the Hodge-Riemann relations.

One of the original approaches used by Huh and Katz to extend their work to non-representable matroids was to try proving a tropical version of the Hodge index theorem for surfaces. However, there are counterexamples to any naïve formulation of such a result (see, e.g., 4, §5.6]), and the situation appears quite delicate - it is unclear what the hypotheses for a tropical Hodge index theorem should be and how to reduce the desired inequalities to this special case. 
So instead, inspired by the work of McMullen and of Fleming and Karu on Hodge theory for simple polytopes [16, 22, Adiprasito, Huh, and Katz developed a completely new method for attacking the general Rota-Welsh conjecture.

In both the realizable case from [18] and the general case from [1], one needs only a very special case of the Hodge-Riemann relations to deduce log-concavity of the coefficients of $\bar{\chi}_{M}(t) 16$ And Poincaré duality and the hard Lefschetz theorem for Chow rings of matroids are not needed at all for this application. So it's reasonable to wonder whether Theorem 5.1 is overkill if one just wants a proof of the RotaWelsh conjecture. It seems that in practice, Poincaré duality, the hard Lefschetz theorem, and the Hodge-Riemann relations tend to come bundled together in what is sometimes called the Kähler package 17 This is the case, for example, in the algebro-geometric work of de Cataldo and Migliorini [9] and of Cattani [8], in the work of McMullen and of Fleming and Karu on Hodge theory for simple polytopes [16, 22, in the work of Elias and Williamson [13] on Hodge theory for Soergel bimodules, and in Adiprasito, Huh, and Katz.

In the case of simple polytopes and the $g$-conjecture, what is needed is in fact the hard Lefschetz theorem, and not the Hodge-Riemann relations, for the appropriate Chow ring. But again the proof proceeds by establishing the full Kähler package.

One of the important differences between [1] and [16], already mentioned above, is that the intermediate objects in the inductive procedure from [1, obtained by applying flips to Bergman fans of matroids, are no longer themselves Bergman fans of matroids (whereas in [16] all of the simplicial fans which appear come from simple polytopes). Another important difference is that in the polytope case, one is working with $n$-dimensional fans in $\mathbb{R}^{n}$, whereas in the matroid case one is working with $d$-dimensional fans in $\mathbb{R}^{n}$, where $d<n$ except in the trivial (but important) case of the $n$-dimensional permutohedral fan. In both the polytope and matroid situations the fan in question defines an $n$-dimensional toric variety, but the toric variety is projective in the polytope case and noncomplete in the matroid case. As mentioned above in \$5.7, the "miracle" in the matroid case is that the Chow ring of the $n$-dimensional noncomplete toric variety $X\left(\Sigma_{M}\right)$ behaves as if it were the Chow ring of a $d$-dimensional smooth projective variety; in particular, it satisfies Poincaré duality, the hard Lefschetz theorem, and Hodge-Riemann relations of "formal" dimension $d$.

5.10. Whitney numbers of the second kind. The Whitney numbers of the second kind $W_{k}(M)$ (cf. 4.5 ) are much less tractable than their first-kind counterparts. In particular, the log-concavity conjecture for them remains wide open. However, there has been recent progress by Huh and Wang [19] concerning a related conjecture, the so-called top-heavy conjecture of Dowling and Wilson:

Conjecture 5.6. Let $M$ be a matroid of rank $r$. Then for all $k<r / 2$, we have $W_{k}(M) \leq W_{r-k}(M)$.

\footnotetext{
${ }^{16}$ Presumably one can use the general Hodge-Riemann relations to deduce other combinatorial facts of interest about matroids!

${ }^{17}$ Note that when some algebraic geometers refer to the Kähler package, they include additional results, such as the Lefschetz hyperplane theorem or Künneth formula, which are not part of [1].
} 
In analogy with the work of Huh and Katz, Huh and Wang prove:

Theorem 5.7 (Huh and Wang, 2016). For all matroids $M$ representable over some field $k$ :

(1) The first half of the sequence of Whitney numbers of the second kind is unimodal, i.e., $W_{1}(M) \leq W_{2}(M) \leq \cdots \leq W_{\lfloor r / 2\rfloor}(M)$.

(2) Conjecture 5.6 is true.

The following corollary is a generalization of the de Bruijn-Erdös theorem that every noncollinear set of points $E$ in a projective plane determines at least $|E|$ lines:

Corollary 5.8. Let $V$ be a d-dimensional vector space over a field, and let $E$ be a subset which spans $V$. Then (in the partially ordered set of subspaces spanned by subsets of $E)$, there are at least as many $(d-k)$-dimensional subspaces as there are $k$-dimensional subspaces, for every $k \leq d / 2$.

We will content ourselves with just a couple of general remarks concerning the proof of Theorem 5.7. Unlike the Rota-Welsh situation of Whitney numbers of the first kind, the projective algebraic variety $Y_{M}^{\prime}$ which one associates to $M$ in this case is highly singular; thus instead of invoking the Kähler package for smooth projective varieties, Huh and Wang have to use analogous but much harder results about intersection cohomology. Specifically, they require the Bernstein-BeilinsonDeligne-Gabber decomposition theorem for intersection complexe 18 and the hard Lefschetz theorem for $\ell$-adic intersection cohomology of projective varieties.

It is tempting to fantasize about a proof of Conjecture 5.6 along the lines of [1. One of many significant challenges in this direction would be to construct a combinatorial model for intersection cohomology of the variety $Y_{M}^{\prime}$.

\section{ACKNOWLEDGMEnTs}

The author thanks Karim Adiprasito, Eric Katz, and June Huh for numerous helpful discussions and corrections, and Bruce Sagan and an anonymous referee for additional useful proofreading.

\section{AbOut THE AUTHOR}

Matthew Baker is professor of mathematics at Georgia Institute of Technology and is an AMS Fellow. His research interests include arithmetic geometry, algebraic combinatorics, tropical and non-Archimedean geometry, and arithmetic dynamics.

\section{REFERENCES}

[1] Karim Adiprasito, June Huh, and Eric Katz, Hodge theory for combinatorial geometries, Preprint. Available at arxiv:math.C0/1511.02888, 61 pages, 2015.

[2] Karim Adiprasito, June Huh, and Eric Katz, Hodge theory of matroids, Notices Amer. Math. Soc. 64 (2017), no. 1, 26-30, DOI 10.1090/noti1463. MR3586249

[3] Christos A. Athanasiadis, Characteristic polynomials of subspace arrangements and finite fields, Adv. Math. 122 (1996), no. 2, 193-233, DOI 10.1006/aima.1996.0059. MR.1409420

[4] Farhad Babaee and June Huh, A tropical approach to the strongly positive Hodge conjecture, To appear in Duke Math. J. Preprint available at arxiv:math. AG/1502.00299, 50 pages, 2015.

[5] Anders Björner, Shellable and Cohen-Macaulay partially ordered sets, Trans. Amer. Math. Soc. 260 (1980), no. 1, 159-183, DOI 10.2307/1999881. MR570784

\footnotetext{
${ }^{18}$ See 10 for an overview of the decomposition theorem and its many applications.
} 
[6] Anders Björner, The homology and shellability of matroids and geometric lattices, Matroid applications, Encyclopedia Math. Appl., vol. 40, Cambridge Univ. Press, Cambridge, 1992, pp. 226-283, DOI 10.1017/CBO9780511662041.008. MR.1165544

[7] Tom Brylawski, The broken-circuit complex, Trans. Amer. Math. Soc. 234 (1977), no. 2, 417-433, DOI 10.2307/1997928. MR468931

[8] Eduardo Cattani, Mixed Lefschetz theorems and Hodge-Riemann bilinear relations, Int. Math. Res. Not. IMRN 10 (2008), Art. ID rnn025, 20, DOI 10.1093/imrn/rnn025. MR2429243

[9] Mark Andrea A. de Cataldo and Luca Migliorini, The hard Lefschetz theorem and the topology of semismall maps (English, with English and French summaries), Ann. Sci. École Norm. Sup. (4) 35 (2002), no. 5, 759-772, DOI 10.1016/S0012-9593(02)01108-4. MR1951443

[10] Mark Andrea A. de Cataldo and Luca Migliorini, The decomposition theorem, perverse sheaves and the topology of algebraic maps, Bull. Amer. Math. Soc. (N.S.) 46 (2009), no. 4, 535-633, DOI 10.1090/S0273-0979-09-01260-9. MR2525735

[11] C. De Concini and C. Procesi, Wonderful models of subspace arrangements, Selecta Math. (N.S.) 1 (1995), no. 3, 459-494, DOI 10.1007/BF01589496. MR.1366622

[12] Graham Denham, Toric and tropical compactifications of hyperplane complements (English, with English and French summaries), Ann. Fac. Sci. Toulouse Math. (6) 23 (2014), no. 2, 297-333, DOI 10.5802/afst.1408. MR3205595

[13] Ben Elias and Geordie Williamson, The Hodge theory of Soergel bimodules, Ann. of Math. (2) 180 (2014), no. 3, 1089-1136, DOI 10.4007/annals.2014.180.3.6. MR 3245013

[14] Eva Maria Feichtner and Sergey Yuzvinsky, Chow rings of toric varieties defined by atomic lattices, Invent. Math. 155 (2004), no. 3, 515-536, DOI 10.1007/s00222-003-03272. MR2038195

[15] Alex Fink, Tropical cycles and Chow polytopes, Beitr. Algebra Geom. 54 (2013), no. 1, 13-40, DOI 10.1007/s13366-012-0122-6. MR.3027663

[16] Balin Fleming and Kalle Karu, Hard Lefschetz theorem for simple polytopes, J. Algebraic Combin. 32 (2010), no. 2, 227-239, DOI 10.1007/s10801-009-0212-1. MR2661416

[17] June Huh, Milnor numbers of projective hypersurfaces and the chromatic polynomial of graphs, J. Amer. Math. Soc. 25 (2012), no. 3, 907-927, DOI 10.1090/S0894-0347-2012-007310. MR2904577

[18] June Huh and Eric Katz, Log-concavity of characteristic polynomials and the Bergman fan of matroids, Math. Ann. 354 (2012), no. 3, 1103-1116, DOI 10.1007/s00208-011-0777-6. MR2983081

[19] June Huh and Botong Wang, Enumeration of points, lines, planes etc., To appear in Acta Mathematica. Preprint available at arxiv:math.C0/1609.05484, 17 pages, 2016.

[20] Eric Katz, Matroid theory for algebraic geometers, Non-Archimedean and Tropical Geometry, Springer, 2016, pp. 435-517.

[21] Matthias Lenz, The $f$-vector of a representable-matroid complex is log-concave, Adv. in Appl. Math. 51 (2013), no. 5, 543-545, DOI 10.1016/j.aam.2013.07.001. MR3118543

[22] Peter McMullen, On simple polytopes, Invent. Math. 113 (1993), no. 2, 419-444, DOI 10.1007/BF01244313. MR1228132

[23] Peter Nelson, Almost all matroids are non-representable, Preprint. Available at arxiv:math.CO/1605.04288, 4 pages, 2016.

[24] Peter Orlik and Louis Solomon, Combinatorics and topology of complements of hyperplanes, Invent. Math. 56 (1980), no. 2, 167-189, DOI 10.1007/BF01392549. MR558866

[25] James G. Oxley, Matroid theory, Oxford Science Publications, The Clarendon Press, Oxford University Press, New York, 1992. MR 1207587

[26] Adrien Saumard and Jon A. Wellner, Log-concavity and strong log-concavity: a review, Stat. Surv. 8 (2014), 45-114, DOI 10.1214/14-SS107. MR.3290441

[27] Richard P. Stanley, Log-concave and unimodal sequences in algebra, combinatorics, and geometry, Graph theory and its applications: East and West (Jinan, 1986), Ann. New York Acad. Sci., vol. 576, New York Acad. Sci., New York, 1989, pp. 500-535, DOI 10.1111/j.17496632.1989.tb16434.x. MR1110850

[28] D. J. A. Welsh, Matroid theory, Academic Press [Harcourt Brace Jovanovich, Publishers], London-New York, 1976. L. M. S. Monographs, No. 8. MR0427112

[29] Neil White (ed.), Combinatorial geometries, Encyclopedia of Mathematics and its Applications, vol. 29, Cambridge University Press, Cambridge, 1987. MR921064 
[30] Günter M. Ziegler, Lectures on polytopes, Graduate Texts in Mathematics, vol. 152, SpringerVerlag, New York, 1995. MR,1311028

School of Mathematics, Georgia Institute of Technology, Atlanta GA 30332-0160, $\mathrm{USA}$

E-mail address: mbaker@math.gatech.edu 